\title{
Clinical Efficacy and Imaging Analysis for the Surgical Treatment of Thoracolumbar Infections in Elderly Patients
}

\section{Yanlong Zhong}

Department of Orthopedics, the First Affiliated Hospital of Nanchang University

\section{Benyu Tang}

Department of Orthopedics, the First Affiliated Hospital of Nanchang University

Qi Lai

Department of Orthopedics, the First Affiliated Hospital of Nanchang University

\section{Yonghong Sheng}

Department of Orthopedics, the First Affiliated Hospital of Nanchang University

\section{Chao Li}

Department of Orthopedics, the First Affiliated Hospital of Nanchang University

Jia Guo

Department of Orthopedics, the First Affiliated Hospital of Nanchang University

\section{Shiwei Luo}

Department of Orthopedics, the First Affiliated Hospital of Nanchang University

\section{Haoqun Yao}

Department of Orthopedics, the First Affiliated Hospital of Nanchang University

\section{Zongmiao Wan ( $\nabla$ zongmia02008@163.com )}

Department of Orthopedics, the First Affiliated Hospital of Nanchang University

\section{Research Article}

Keywords: Spinal infection, Surgical treatment, pyogenic spondylitis, tuberculous spondylitis, surgical outcome

Posted Date: March 23rd, 2021

DOI: https://doi.org/10.21203/rs.3.rs-269772/v1

License: (1) (1) This work is licensed under a Creative Commons Attribution 4.0 International License. Read Full License 


\section{Abstract}

\section{Background}

Few reports have been conducted to comparing surgical results and safety evaluations between the different types of infections in geriatric patients. The aim of this study is to investigate the safety and efficacy of surgical treatment for thoracolumbar infections in elderly patients.

\section{Methods}

21 patients with pyogenic spondylodiscitis (PS) and 26 patients with tuberculous spondylodiscitis (TS) were enrolled in the study. All patients were treated using one-stage posterior debridement, decompression, and pedicle screw fixation. Comparison of operative safety parameters between the two groups. Clinical efficacy was evaluated using visual analog scale (VAS) score, the American Spinal Injury Association (ASIA) grade, the short form (SF)-36 survey and Oswestry disability index (ODI) to determine patient quality of life pre- and post-operatively.

\section{Results}

Hospitalisation and intensive care unit duration in the PS group were significantly shorter than in the TS group $(P<0.05)$. The total incidence of post-operative complications for both groups was $44.7 \%$. More complications occurred in the TS group, but the difference was not significant. The VAS and SF-36 scores (physical component) were significantly better in the PS group six months post-operatively, and the SF-36 (mental component) scores were significantly better in the PS group at the one-year follow-up.

Neurological status in both groups improved post-operatively, and $83 \%$ of the patients were satisfied with the results of their operation. Imaging results showed that bone graft fusion improved in both groups at six months, one year and at the final follow-up.

\section{Conclusion}

Surgical treatment can provide satisfactory clinical and imaging results for thoracolumbar infection in patients over 65 years old. Although the incidence of peri-operative complications in the elderly is high, it is controllable and surgical treatment remains a safe and effective measure.

\section{Background}

With the aging of the population and the improvement of medical diagnosis, the number of elderly patients diagnosed with infectious spondylitis has increased in the past few decades[1, 2]. Infectious spondylitis is a special biological type of infectious disease, which affects the vertebral body, intervertebral disc, and adjacent perivertebral soft tissue[3,4]. A variety of pathogens can cause spinal infections, with common bacteria and Mycobacterium tuberculosis being the primary pathogens[5]. Pyogenic spondylodiscitis (PS) is an infectious spondylitis caused by common bacteria, while spondylitis caused by M. tuberculosis is termed tuberculous spondylodiscitis (TS) [6]. 
For elderly patients with decreased immune resistance and poor physical functioning, conservative treatments such as immobilisation and antibiotic administration should be the first choice [7, 8]. However, these treatments require prolonged bed rest, which can lead to complications including urinary system infections, lower limb deep venous thrombosis and pulmonary infection, all of which may seriously affect patient quality of life [9].Therefore, to optimally target the pathology of the disease and facilitate early mobilisation in the elderly, a quick, effective, and safe approach is needed. Surgical decompression is the preferred treatment when neurological deficits or progressive deformity occurs during disease progression [10-13].

Currently, the surgical treatment for infectious spondylitis is divided into the following four techniques, including posterior stabilization alone, anterior only, anterior combined, or posterior combined[14]. Several authors have reported the results for these surgical techniques and have confirmed that the single posterior approach is well established in patients with infectious spondylitis $[15,16]$. However, there are few reports comparing surgical results and safety evaluations between the different types of infections in geriatric patients $[9,17]$. In this study, we compared the clinical and radiological results of one-stage posterior surgery for elderly patients with PS or TS at a single institution, and evaluated the efficacy and safety of the surgical treatment.

\section{Materials And Methods}

\section{Patient characteristics}

We used our hospital electronic medical record system (Haitai® 3.0, Nanjing, China) and image archiving and communication system (Carestream ${ }^{\circledR}$, Jiangxi, China) to retrospectively analyse the data of 59 elderly patients with infectious spondylitis who underwent one-stage posterior debridement, decompression, and pedicle screw fixation at our department from January 2012 to September 2017. The inclusion criteria were: (1) patients who were diagnosed with suppurative or tuberculous spondylitis and had received surgical treatment, (2) 65 years or older at the time of their operation, and (3) had a postoperative follow-up of $\geq 1$ year. The exclusion criteria were: (1) incomplete medical records or imaging data, and (2) patients without telephones or out-patient follow-up. The diagnosis of infectious spondylitis was based on clinical symptoms, laboratory results and radiological evidence. Histopathology following debridement was confirmed. Patients were divided into a PS or TS group according to the pathological results (Table 1). 
Table 1

Demographic data for the pyogenic spondylodiscitis (PS) and tuberculous spondylodiscitis (TS) groups

\begin{tabular}{|c|c|c|c|}
\hline Characteristic & PS & TS & Total \\
\hline Number of cases & 21 & 26 & 47 \\
\hline Age in years, median (range) & $75(67-82)$ & $76(66-80)$ & $76(66-82)$ \\
\hline \multicolumn{4}{|l|}{ Sex, number } \\
\hline Female & 11 & 14 & 25 \\
\hline Male & 10 & 12 & 22 \\
\hline BMI, median (range) & $19.7(17.1-25.8)$ & $20.6(19.6-28.3)$ & $20.4(17.1-28.3)$ \\
\hline Smoking history, number & 11 & 9 & 20 \\
\hline Months of follow-up, median (range) & $24(13-45)$ & $29(12-68)$ & $26(12-68)$ \\
\hline \multicolumn{4}{|l|}{ Level of infection, number } \\
\hline Thoracic & 4 & 20 & 24 \\
\hline Lumbar & 15 & 4 & 19 \\
\hline Thoracic + Lumbar & 2 & 2 & 4 \\
\hline \multicolumn{4}{|l|}{ Co-morbidities, number } \\
\hline Cardiovascular disease & 7 & 4 & 11 \\
\hline Diabetes mellitus & 5 & 8 & 13 \\
\hline COPD & 4 & 7 & 11 \\
\hline Hepatitis & 3 & 4 & 7 \\
\hline None of the above diseases & 2 & 3 & 5 \\
\hline \multicolumn{4}{|l|}{ BMI: body mass index } \\
\hline COPD: chronic obstructive pulmonar & lisease & & \\
\hline
\end{tabular}

\section{Peri-operative safety evaluations}

Two groups of indexes related to surgical safety were reviewed and recorded in the medical records system including hospital-stay duration, operation time, blood loss, suspended red blood cell transfusion (peri-operative period) and duration in the intensive care unit (ICU). Common post-operative complications were closely observed and included incision infection, pulmonary infection, venous thrombosis, heart failure and liver function damage.

\section{Clinical efficacy evaluations}


Clinical outcomes were assessed using analogue scale (VAS) to evaluate back pain. The Oswestry disability index (ODI) was used to quantify disability associated with lower back pain, and the short form (SF)-36 concise health status questionnaire was used to determine patient quality of life both preoperatively and post-operatively (at the three-month, six-month, one-year, and final follow-up time points). The American Spinal Injury Association (ASIA) scale was used to compare the state of the nervous system pre- and post-operatively in the two groups. Surgical outcome satisfaction was assessed using the modified MacNab criteria [18] at the final follow-up(>12months).

\section{Radiographic measurements}

Two independent observers (both senior spinal surgeons), who did not participate in the operations, performed the imaging analyses, Digital $x$-ray images were analysed six months and one year following the operations and during the final out-patient visit to evaluate bone fusion status. The fusion status was classified according to the modified Bridwell criteria [19].

To evaluate the effect of stress on internal fixation and the maintenance of sagittal balance, we measured the Cobb angle using the cephalic and caudal endplates of the infected vertebral body. The changes in the sagittal Cobb angle were calculated pre-operatively, immediately after the operation, and at the final follow-up.

\section{Statistical analysis}

Clinical outcomes and radiographic data are presented as means \pm standard deviations and were compared between the PS and TS groups using the Student t-test. The chi-square test was used to compare the incidence of complications and satisfaction levels between the PS and TS groups. The ranksum test was used to compare the early fusion rates. $P<0.05$ was considered to indicate statistical significance. All analyses were performed using the SPSS 16.0 statistical software package (Statistical Software for Social Sciences, Chicago, IL, USA).

\section{Results}

Of the 59 eligible patients, 12 were excluded from the study. Nine patients were lost during out-patient or telephone follow-up, and three died of other diseases during the follow-up period. In total, 47 patients were included for analysis-21 patients with PS and 26 patients with TS.

\section{Patient demographic data}

There were no significant differences in age, sex, body mass index, smoking history, or duration of the follow-up time between the PS and TS groups. Details of the infections and co-morbidities are shown in Table 1.

\section{Clinical outcomes of the surgical treatment}


The hospitalisation and ICU durations in the PS group were significantly shorter than those in TS group $(P<0.05)$. In addition, the amount of intraoperative blood loss and suspended erythrocyte transfusion, and the operation time in the TS group were higher than the PS group, but there was no significant difference between the two groups $(P>0.05)$. A complete listing of the clinical variables for both groups is provided in Table 2 .

Table 2

Comparison of clinical and radiological parameters in the PS and TS groups

\begin{tabular}{|llll|}
\hline Parameters & PS & TS & P-Value \\
\hline clinical & & & \\
\hline Length of hospitalisation (days) & $32.48 \pm 8.07$ & $40.88 \pm 13.52$ & $0.007^{\star}$ \\
\hline Blood loss during operation (ml) & $526.19 \pm 197.24$ & $634.62 \pm 269.36$ & 0.065 \\
\hline Infusion of suspended red blood cells(u) & $1.29 \pm 1.42$ & $1.57 \pm 1.84$ & 0.536 \\
\hline Operation time (minutes) & $172.38 \pm 42.46$ & $190.85 \pm 43.86$ & 0.169 \\
\hline Intensive care unit length of stay (days) & $2.24 \pm 1.04$ & $3.31 \pm 1.43$ & $0.003^{\star}$ \\
\hline radiographic & $24.54 \pm 5.05$ & $26.62 \pm 4.55$ & 0.072 \\
\hline Pre-operative $\left(^{\circ}\right)$ & $15.59 \pm 5.32$ & $15.73 \pm 3.97$ & 0.459 \\
\hline Post-operative $\left(^{\circ}\right)$ & $18.48 \pm 5.28$ & $18.78 \pm 3.80$ & 0.409 \\
\hline Final follow-up $\left(^{\circ}\right)$ & $2.89 \pm 1.42$ & $3.05 \pm 1.99$ & 0.376 \\
\hline Loss angle of correction $\left({ }^{\circ}\right)$ & $24.54 \pm 5.05$ & $26.62 \pm 4.55$ & 0.072 \\
\hline PS:pyogenic spondylodiscitis; TS:tuberculous spondylodiscitis; ICU:intensive care unit. \\
\hline The “*” represents significant difference (P<0.05). & & \\
\hline
\end{tabular}

\section{Post-operative complications}

The total incidence of postoperative complications in both groups was $44.7 \%$. Only the number of patients with complications was counted, and there was no overlap if the same patient experienced multiple complication. The complication rate was $42.9 \%$ in the PS group and $46.1 \%$ in the TS group. There was no significant difference between the two groups $(P>0.05)$. In the $P S$ group, two patients experienced cerebrospinal fluid leakage post-operatively. The drainage tubes were removed, re-sutured and healed without serious consequences after intensive anti-infective treatment. One patient developed an infection in the iliac bone removal area, which healed after changing the dressing and the administration of long-term antibiotics. One patient was followed up for 6 months with a pedicle screw fracture without any discomfort. One patient showed weakness during right ankle extension. However, the symptoms gradually improved within six months post-operatively. Pulmonary infection occurred in two cases and deep venous thrombosis occurred in one case post-operatively. In TS group, cerebrospinal fluid 
leakage was found in two cases. The tube was extubated 48 hours later; however, there was still cerebrospinal fluid outflow from the orifice of the drainage tube, which was improved after silk blood suturing. One case developed a deep wound infection, which healed following debridement, suturing and active anti-infective treatment. One patient was found to have pedicle screw dislocation penetrating the spinal canal, but there were no neurological symptoms. Pulmonary infection occurred in three cases. No other serious complications were caused by active anti-inflammatory treatment. One case developed heart failure, which was cured after proper treatment. Liver damage occurred in two patients, which improved following treatment. One patient experienced a local recurrence of tuberculosis infection, which was controlled after secondary debridement. Deep venous thrombosis of the lower extremities was found in one patient post-operatively and improved after treatment. Lacunar cerebral infarction occurred in one patient, but there was no dysfunction.

\section{Related outcomes of the clinical efficacy of surgical treatment}

The neurological status of the patients is shown in Table 3. All the patients in both groups had different degrees of neurological symptoms prior to their operations. According to the ASIA classification, there were eight cases of ASIA grade D, 12 cases of grade $C$, and one case of grade $B$ in the PS group, and nine cases of ASIA grade D, 15 cases of grade $C$, and two cases of grade B in the TS group. At the final followup, there were three cases of ASIA grade D and 18 cases of grade $E$ in the PS group. In the TS group, there were four cases of ASIA grade D and 22 cases of grade $E$. There were no intra-operative nerve injuries in either group. Our results showed that the neurological symptoms of all the patients had improved postoperatively.

Table 3

Neurological recovery according to the American Spinal Injury Association (ASIA) impairment scale.

\begin{tabular}{|c|c|c|c|c|c|c|c|c|c|c|c|}
\hline \multirow[t]{2}{*}{ Pre-operation } & \multirow[t]{2}{*}{ Group PS/TS } & \multicolumn{5}{|c|}{ Final follow-up in the PS group } & \multicolumn{5}{|c|}{ Final follow-up in the TS group } \\
\hline & & A & B & C & $\mathrm{D}$ & E & A & B & $\mathrm{C}$ & $\mathrm{D}$ & $\mathrm{E}$ \\
\hline A & $0 / 0$ & & & & & & & & & & \\
\hline B & $1 / 2$ & & & & 1 & & & & & 1 & 1 \\
\hline C & $12 / 15$ & & & & 2 & 10 & & & & 3 & 12 \\
\hline D & $8 / 9$ & & & & & 8 & & & & & 9 \\
\hline \multicolumn{12}{|l|}{ E } \\
\hline \multicolumn{12}{|c|}{ PS: pyogenic spondylodiscitis; TS: tuberculous spondylodiscitis } \\
\hline The final follo & up time is more & nan & & & & & & & & & \\
\hline
\end{tabular}

During follow-up period, the VAS, ODI, and the mental component summary (MCS) score and physical component summary (PCS) score of the SF-36 in the PS and TS groups were significantly improved post- 
operatively (Fig. 1). At the six-month follow-up, the VAS score of the PS group $(2.15 \pm 0.61)$ was significantly $(P<0.05)$ better than the TS group $(2.52 \pm 0.51)$ (Fig. 1a). For the ODI and PCS scores, the TS group had slightly worse scores than the PS group(Fig. 1b,c). However, the average PCS score in the PS group was $48.19 \pm 5.63$, which was significantly better than the TS group $(45.42 \pm 4.87)$ at the six-month follow-up $(\mathrm{P}<0.05)$ (Fig. 1C). One year post-operatively, the average MCS score in the PS group was 50.19 \pm 4.07 , which was better than the TS group (45.62 \pm 5.22$)$, and the differences were statistically significant $(P<0.05)$ (Fig. 1d). Approximately $83 \%$ of patients reported satisfactory results based on the modified MacNab standard.

\section{Imaging outcomes of surgical treatment}

The average Cobb angle measurements of the PS group pre-operatively, post-operatively, and at the final follow-up were $24.54 \pm 5.05^{\circ}, 15.59 \pm 5.32^{\circ}$, and $18.48 \pm 5.28$, respectively. For the TS group, the average pre-operative, post-operative, and final follow-up Cobb angle measurements were $26.62 \pm 4.55^{\circ}, 15.73 \pm$ $3.97^{\circ}$, and $18.78 \pm 3.80^{\circ}$, respectively. At the final follow-up, the mean loss angle of correction was $2.89 \pm$ $1.42^{\circ}$ and $3.05 \pm 1.99^{\circ}$ in the PS and TS groups, respectively. No statistical difference between the two groups was found. The Cobb angle was corrected operatively in both groups, but there was no statistical significance between the two groups at the pre-operative, post-operative or final follow-up periods. (Table 2)

At the six-month, one-year, and final follow-up time points, the fusion rates of the PS group were $66.7 \%$, $85.7 \%$, and $90.5 \%$, respectively, which were not significantly different from the fusion rates of the TS group (73.1\%, 88.5\%, and $92.4 \%$, respectively) (Fig. 2).

\section{Clinical presentations}

\section{Case 1}

A 70-year-old female complained of severe lower back pain, with diabetes. An x-ray showed narrowing of the intervertebral disc space in T12-L1 (Fig. 3a) and a CT scan showed bone destruction in T12-L1 with obvious hyper osteogeny and sclerosis (Fig. 3b, c). A lateral magnetic resonance imaging (MRI) on T2 showed high signal intensity of the T12-L1 vertebral body, loss of the intervertebral disc and irregular destruction of the vertebral endplate boundary (Fig. 3d). She received conservative treatment for one month and, although the pain lessened, the pain and inflammation remained. As a result, she underwent one-stage posterior operation. Following the operation, her severe lower back pain was significantly improved (Fig. 3e). Lateral x-ray films taken three months, six months, one year, two years and at the final follow-up showed that the physiological curvature and intervertebral height of the lumbar vertebrae had gradually recovered, the bone graft had gradually fused and she no longer experienced back pain (Fig. 3fj).

\section{Case 2}


A 67-year-old male complained of severe lower back pain with neurological symptoms. He suffered from tuberculosis and high blood pressure. A CT scan and MRI showed bone destruction, para-vertebral abscess formation and spinal cord compression in T6- T7 (Fig. 4a-d). Following three months of medication and brace treatment, he underwent operation to reduce the severity of the back pain (Fig. 4e). The specimen extracted during the operation was positive for $M$. tuberculosis. Lateral x-ray films at six months, one year and two years post-operation showed that the bone graft had gradually fused (Fig. 4fh).

\section{Discussion}

The purpose of the surgical treatment for thoracolumbar infectious spondylitis in the elderly is to facilitate early mobilization after the operation. One-stage posterior focus debridement, bone graft fusion, and internal fixation are widely accepted methods for the treatment of spinal infections in the elderly [9], [20]. For elderly patients in poor health and with poor surgical tolerance, this method can effectively improve the safety of the operation. Our results show that the surgical treatment of infectious spondylitis was effective, enabling $91 \%$ of the patients who received surgical treatment to walk independently during the follow-up period. Although we only included patients over 65 years of age, our results are consistent with previous reports [21-24].

In our study, we found no significant differences between the PS and TS groups for mean blood loss and the operation duration. However, hospitalization and ICU stay duration in the PS group were significantly shorter than in the TS group. Okada et al. [25] reported similar results. TS is a long-term chronic wasting disease that is usually associated with systemic diseases such as tuberculosis, and patients with this condition are more likely to develop anaesthesia-related complications, which may help to explain this phenomenon [26]. Thus, a post-operative stay in the ICU and delays in the recovery process would be inevitable. In particular, the onset of tuberculosis is typically unknown, the early symptoms are atypical and it takes longer to diagnose, which partially explains why the hospitalisation duration for patients in the TS group was longer than in the PS group. In our study, $44.7 \%$ of patients had post-operative complications. In a related study of elderly patients with spinal tuberculosis, Luo et al. [27] reported a $97 \%$ incidence of complications, which may be due to the absence of statistics on the complications of waterelectrolyte imbalance. However, we did not find significant differences in complication rates between the two groups.

In terms of the clinical follow-up results, the VAS, ODI and the MCS and PCS scores of the SF-36 for the TS and PS groups were significantly improved from the pre-operative evaluation to the final follow-up. However, the VAS score for lower back pain in the TS group was significantly lower than that in PS group six months post-operatively $(P<0.05)$ (Fig. 1). Okada et al. [21] reported that the duration of achieving a CRP-negative result in a TS group was longer than for a PS group (105.9 \pm 16.3 vs $52.6 \pm 20.2$ days), and patients with tuberculous spondylitis experienced more severe inflammatory stimulation within a short period following an operation. Therefore, before inflammation subsides, PCS scores may demonstrate significant differences. Correspondingly, the PCS scores in our TS group were lower than the PS group at 
the six-month follow-up. At the one-year follow-up, the MCS score of the TS group was worse than the PS group.

The literature [28]suggests that tuberculosis is usually associated with poor living standards and social factors, which can more strongly affect the elderly. Therefore, we speculate that surgical trauma has a greater impact on patients with TS than PS; although, the difference may become indistinct over time. Chun Kim et al. [29] reported that at the final follow-up of 485 people, $85 \%$ demonstrated excellent or good results in lumbar fusion in the elderly according to the MacNab criteria. Similarly, approximately $83 \%$ of the patients in our study were satisfied with the post-operative results, indicating that the positive effects of surgical treatment for the elderly should be expected if the operation is safe.

Similar results from other studies [30], [31], [32], [33] have shown that solid bone fusion occurs in over $90 \%$ of cases. Likewise, the solid fusion rates in the PS and TS groups in our study also reached $90.5 \%$ and $92.4 \%$, respectively, at the final follow-up. Although there was no statistical difference between the PS and TS groups, both achieved good fusion rate results. We assumed TS might hamper the maintenance of alignment due to necrotic disease and osteoporosis [21], [27], and it has been reported that TS is more frequently associated with greater deformity than PS [34]. However, we found no significant difference in the correcting angle loss between the PS and TS groups. This is similar to that reported by Okada et al. [21],however, definitive results may require longer follow-up observation times.

There were some limitations in our study. First, the sample sizes in the two groups were small. A sample size of 47 patients is insufficient and was due to the small number of elderly surgical patients. Second, our average follow-up period of 26 months was not enough to observe long-term effects. Finally, our study focused on specific sub-groups and did not make comparisons with conservative treatments in the elderly, which may have led to inaccurate results. Further, multi-centre, randomised, long-term follow-up studies are needed to overcome these issues.

\section{Conclusion}

Operation can effectively improve the neurological functioning of elderly patients, reconstruct spinal stability, improve patient quality of life and provide satisfactory clinical and imaging results for thoracolumbar infection. Although the incidence of peri-operative complications in the elderly is high, it is controllable and surgical treatment remains a safe and effective measure.

\section{Abbreviations}

PS

Pyogenic spondylodiscitis; TS:Tuberculous spondylitis; ASIA:American Spinal Injury Association; VAS:Visual analog scale; ODI:Oswestry disability index; SF-36:Short form-36 health survey questionnaire; PCS:Physical component summary; MCS:Mental component summary; ICU:Intensive Care Unit 


\section{Declarations}

\section{Ethics approval and consent to participate}

This study was approved by the ethical review committee of The First Affiliated Hospital of Nanchang. All procedures were performed in accordance with relevant guidelines. University before the initiation of the study and a signed informed consent form was obtained from each subject.

\section{Consent to publich}

All involved subjects and the authors listed have approved the publication of the manuscript.

\section{Availability of data and materials}

The datasets supporting the conclusions of this article are included within the article. The raw data can be requested from the corresponding author on reasonable request.

\section{Competing interests}

The authors declare that they have no competing interests.

\section{Funding}

The authors would like to gratefully acknowledge the financial support from the Natural Science Foundation of China [grant numbers 81760410,81960408] \and Key R \& D projects of Jiangxi Province Science and Technology Department [grant numbers 20202BBG73033].

\section{Authors' contributions}

$\mathrm{YZ}$ and $\mathrm{BT}$ performed all the researches and wrote the manuscript. QL, YS, CL, JG and SL participated in the collection of researchful data. HY guided the entire process of the research. ZW conceived and designed the study. All authors read and approved the final manuscript.

\section{Acknowledgements}

Not applicable.

\section{References}

1. Jones NS, Anderson DJ, Stiles PJ: Osteomyelitis in a general hospital. A five-year study showing an increase in subacute osteomyelitis. J Bone Joint Surg Br 1987, 69(5):779-783.

2. Emery SE, Chan DP, Woodward HR: Treatment of hematogenous pyogenic vertebral osteomyelitis with anterior debridement and primary bone grafting. Spine (Phila Pa 1976) 1989, 14(3):284-291.

3. Huttner B, Opravil M: [Infectious spondylitis]. Z Rheumatol 2006, 65(1):7-11. 
4. Pintado-García V: Espondilitis infecciosa. Enfermedades Infecciosas Y Microbiología Clínica 2008, 26(8):510-517.

5. Hernández PLM, López MA, Vargas FZ, Paso PGD, Muoz EV: [Spontaneous infectious spondylodiscitis in an internal medicine department: epidemiological and clinical study in 41 cases]. Revista Clínica Espaola 2008, 208(7):347-352.

6. Kim CJ, Song KH, Jeon JH, Park WB, Park SW, Kim HB, Oh MD, Choe KW, Kim NJ: A comparative study of pyogenic and tuberculous spondylodiscitis. Spine (Phila Pa 1976) 2010, 35(21):E10961100.

7. Dimar JR, Carreon LY, Glassman SD, Campbell MJ, Hartman MJ, Johnson JR: Treatment of Pyogenic Vertebral Osteomyelitis With Anterior Debridement and Fusion Followed by Delayed Posterior Spinal Fusion. Spine 2004, 29(3):326-332.

8. Safran O, Rand N, Kaplan L, Sagiv S, Floman Y: Sequential or simultaneous, same-day anterior decompression and posterior stabilization in the management of vertebral osteomyelitis of the lumbar spine. Spine 1998, 23(17):1885-1890.

9. Xu Z, Wang X, Shen X, Luo C, Zeng H, Zhang P, Peng W: Posterior only versus combined posterior and anterior approaches for lower lumbar tuberculous spondylitis with neurological deficits in the aged. Spinal Cord 2015, 53(6):482-487.

10. Chen WH, Jiang LS, Dai LY: Surgical treatment of pyogenic vertebral osteomyelitis with spinal instrumentation. Eur Spine J 2007, 16(9):1307-1316.

11. AG H, JT M, JT N, AJ M: Hematogenous pyogenic spinal infections and their surgical management. Spine 2000, 25(13):1668-1679.

12. Ogden AT, Kaiser MG: Single-stage debridement and instrumentation for pyogenic spinal infections. Neurosurgical FOCUS 2004, 17(6):E5.

13. A Q, Jun P, Jacobs R, Rosenberg WS, Weinstein PR: General principles in the medical and surgical management of spinal infections: a multidisciplinary approach. Neurosurgical Focus 2004, 17(6).

14. Zarghooni K, R? Llinghoff M, Sobottke R, Eysel P: Treatment of spondylodiscitis. International Orthopaedics 2012, 36(2):405-411.

15. Rawall S, Mohan K, Nene A: Posterior approach in thoracolumbar tuberculosis: a clinical and radiological review of 67 operated cases. Musculoskelet Surg 2013, 97(1):67-75.

16. Lu ML, Niu CC, Tsai TT, Fu TS, Chen LH, Chen WJ: Transforaminal lumbar interbody debridement and fusion for the treatment of infective spondylodiscitis in the lumbar spine. Eur Spine J 2015, 24(3):555-560.

17. Kao FC, Tsai TT, Niu CC, Lai PL, Chen LH, Chen WJ: One-stage posterior approaches for treatment of thoracic spinal infection: Transforaminal and costotransversectomy, compared with anterior approach with posterior instrumentation. Medicine (Baltimore) 2017, 96(42):e8352.

18. Faraj AA, Webb JK: Spinal instrumentation for primary pyogenic infection report of 31 patients. Acta Orthop Belg 2000, 66(3):242-247. 
19. Bridwell KH, O'Brien MF, Lenke LG, Baldus C, Blanke K: Posterior spinal fusion supplemented with only allograft bone in paralytic scoliosis. Does it work? Spine (Phila Pa 1976) 1994, 19(23):26582666.

20. Zhang HQ, Li JS, Zhao SS, Shao YX, Liu SH, Gao Q, Lin MZ, Liu JY, Wu JH, Chen J: Surgical management for thoracic spinal tuberculosis in the elderly: posterior only versus combined posterior and anterior approaches. Arch Orthop Trauma Surg 2012, 132(12):1717-1723.

21. Okada Y, Miyamoto H, Uno K, Sumi M: Clinical and radiological outcome of surgery for pyogenic and tuberculous spondylitis: comparisons of surgical techniques and disease types. J Neurosurg Spine 2009, 11(5):620-627.

22. Dimar JR, Carreon LY, Glassman SD, Campbell MJ, Hartman MJ, Johnson JR: Treatment of pyogenic vertebral osteomyelitis with anterior debridement and fusion followed by delayed posterior spinal fusion. Spine (Phila Pa 1976) 2004, 29(3):326-332; discussion 332.

23. Safran O, Rand N, Kaplan L, Sagiv S, Floman Y: Sequential or simultaneous, same-day anterior decompression and posterior stabilization in the management of vertebral osteomyelitis of the lumbar spine. Spine (Phila Pa 1976) 1998, 23(17):1885-1890.

24. Kawamoto E, Wakamatsu M, Fujikake K, Machino A, Kaida T: [Anesthetic considerations for spine surgery in 71 patients with infectious spondylitis: effects of different pathogen either pyogenic or tuberculous on intraoperative blood loss]. Masui 2010, 59(6):711-714.

25. Y O, H M, K U, M S: Clinical and radiological outcome of surgery for pyogenic and tuberculous spondylitis: comparisons of surgical techniques and disease types. Journal of neurosurgery Spine 2009, 11(5):620-627.

26. Kothari M, Shah K, Tikoo A, Nene A: Short to Mid-Term Term Surgical Outcome Study with Posterior Only Approach on Tuberculous Spondylodiscitis in an Elderly Population. Asian Spine J 2016, 10(2):258-266.

27. Luo C, Wang X, Wu P, Ge L, Zhang H, Hu J: Single-stage transpedicular decompression, debridement, posterior instrumentation, and fusion for thoracic tuberculosis with kyphosis and spinal cord compression in aged individuals. Spine J 2016, 16(2):154-162.

28. Blumberg HM, Leonard MK: Musculoskeletal Tuberculosis. Microbiology Spectrum 2017, 5(2).

29. Kim IC, Hur JW, Kwon KY, Lee JJ, Lee HK: The Efficacy and Perioperative Complications Associated with Lumbar Spinal Fusion Surgery, Focusing on Geriatric Patients in the Republic of Korea. Journal of Korean Neurosurgical Society 2013, 54(4):323-328.

30. Fukuta S, Miyamoto K, Masuda T, Hosoe H, Kodama H, Nishimoto H, Sakaeda H, Shimizu K: Twostage (posterior and anterior) surgical treatment using posterior spinal instrumentation for pyogenic and tuberculotic spondylitis. Spine (Phila Pa 1976) 2003, 28(15):E302-308.

31. Fayazi AH, Ludwig SC, Dabbah M, Bryan Butler R, Gelb DE: Preliminary results of staged anterior debridement and reconstruction using titanium mesh cages in the treatment of thoracolumbar vertebral osteomyelitis. Spine J 2004, 4(4):388-395. 
32. Cavusoglu H, Kaya RA, Turkmenoglu ON, Tuncer C, Colak I, Aydin Y: A long-term follow-up study of anterior tibial allografting and instrumentation in the management of thoracolumbar tuberculous spondylitis. J Neurosurg Spine 2008, 8(1):30-38.

33. Korovessis P, Petsinis G, Koureas G, lliopoulos P, Zacharatos S: One-stage combined surgery with mesh cages for treatment of septic spondylitis. Clinical Orthopaedics \& Related Research 2006, 444(\&NA;):51-59.

34. Klockner C, Valencia R: Sagittal alignment after anterior debridement and fusion with or without additional posterior instrumentation in the treatment of pyogenic and tuberculous spondylodiscitis. Spine (Phila Pa 1976) 2003, 28(10):1036-1042.

\section{Figures}
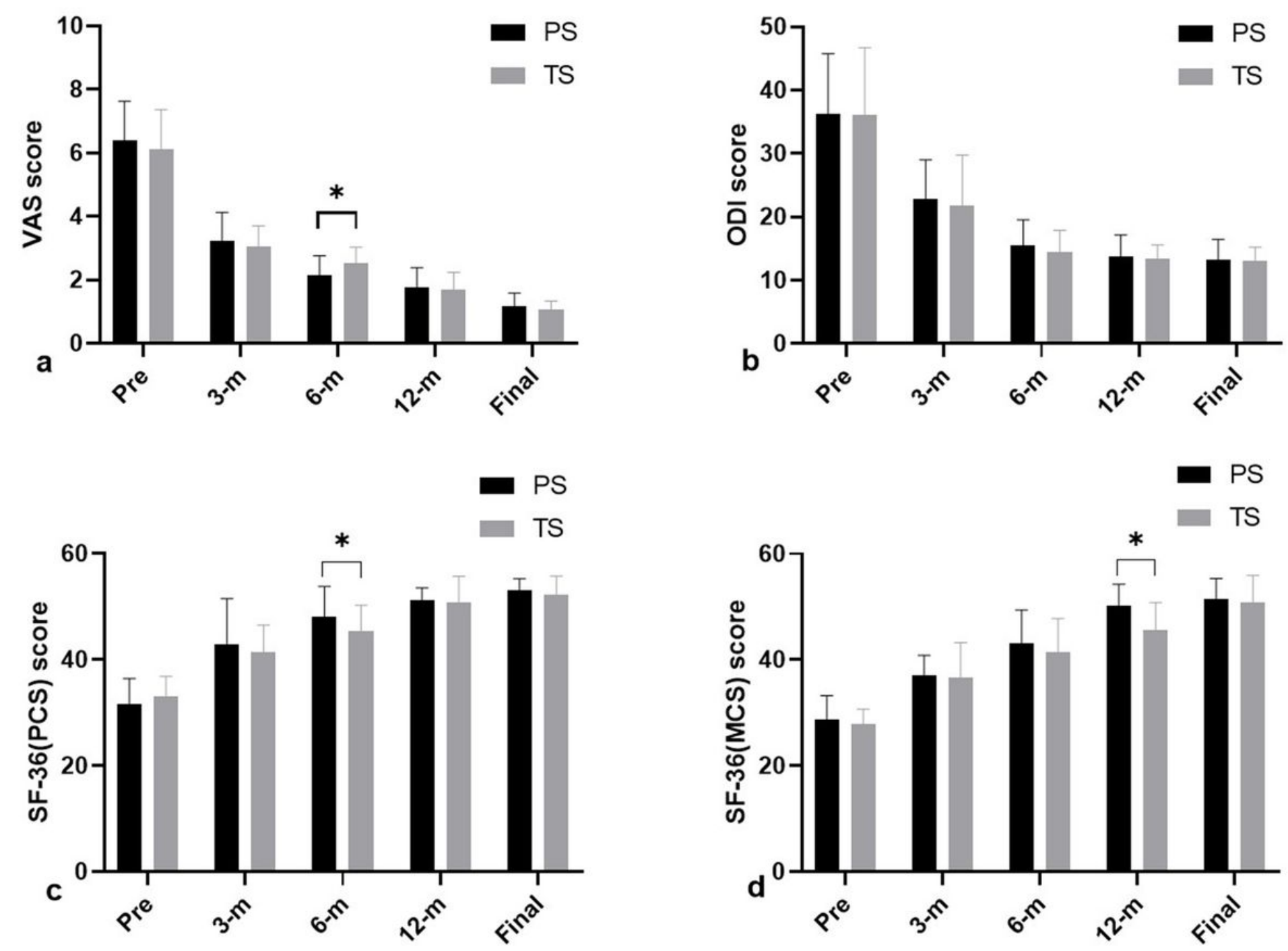

Figure 1 
Outcomes of the clinical efficacy in the two groups as measured by visual analog scale (VAS), Oswestry disability index (ODI), and short form 36 survey ((SF-36) scores at pre-operative, 1-month (1-m), 3-month (3-m), 6-month (6-m), and final follow-up (Final) after operation. The histogram show (a) VAS, (b) ODI, (c) SF-36 (physical component summary ,PCS), (d) SF-36 (mental component summary ,MCS) scores between the pyogenic spondylodiscitis (PS) and tuberculous spondylodiscitis (TS) at different follow-up time points. The " $\star$ " represents significant difference $(P<0.05)$.

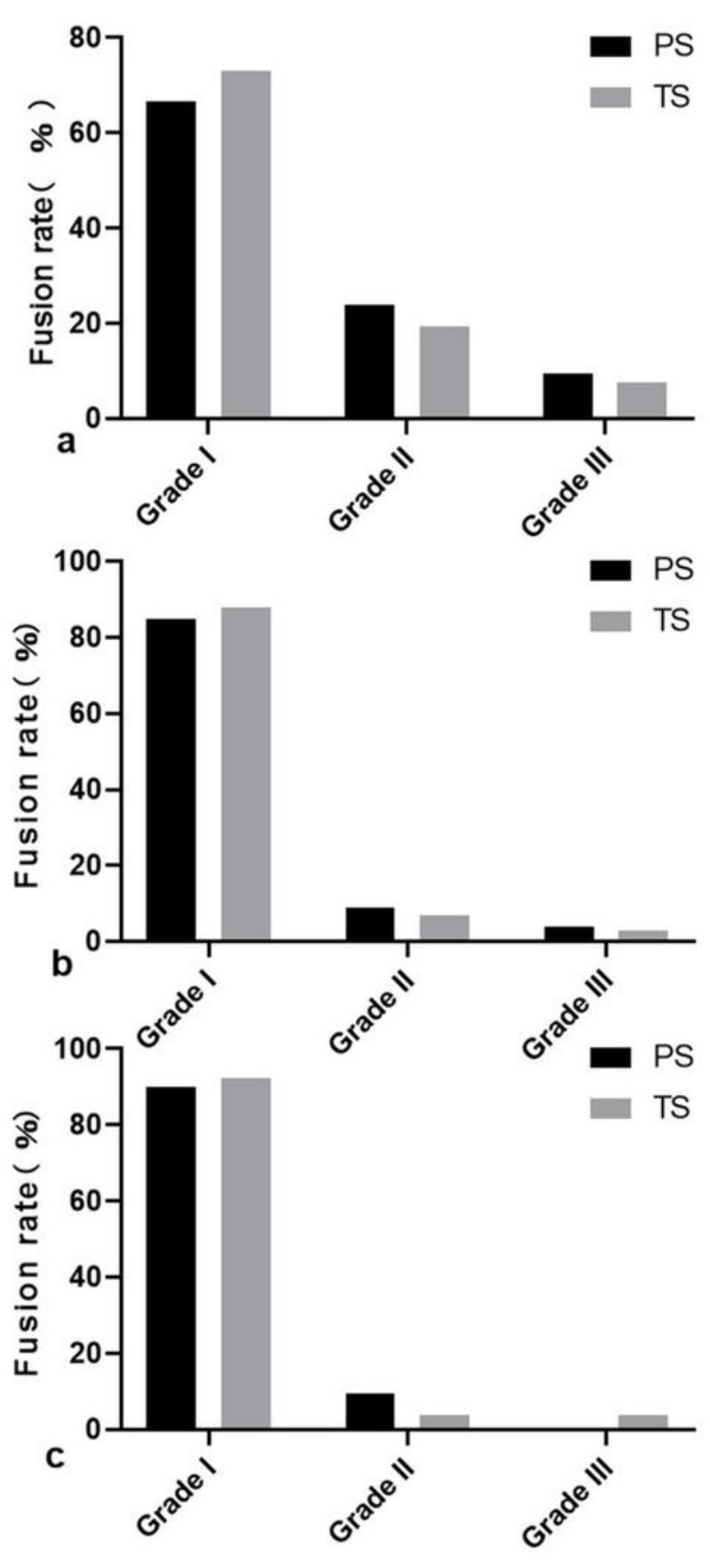

Figure 2 
Post-operative fusion rates in the pyogenic spondylodiscitis (PS) and tuberculous spondylodiscitis (TS) groups at different follow-up time points. Follow-up time at six months (a), at 12 months (b), more than 12 months (c). Fusion rates are shown as Bridwell et al. described interbody fusion rating, with grade 1 was considered solid fusion, which was compared PS and TS groups with interbody fusion using rank sum test.

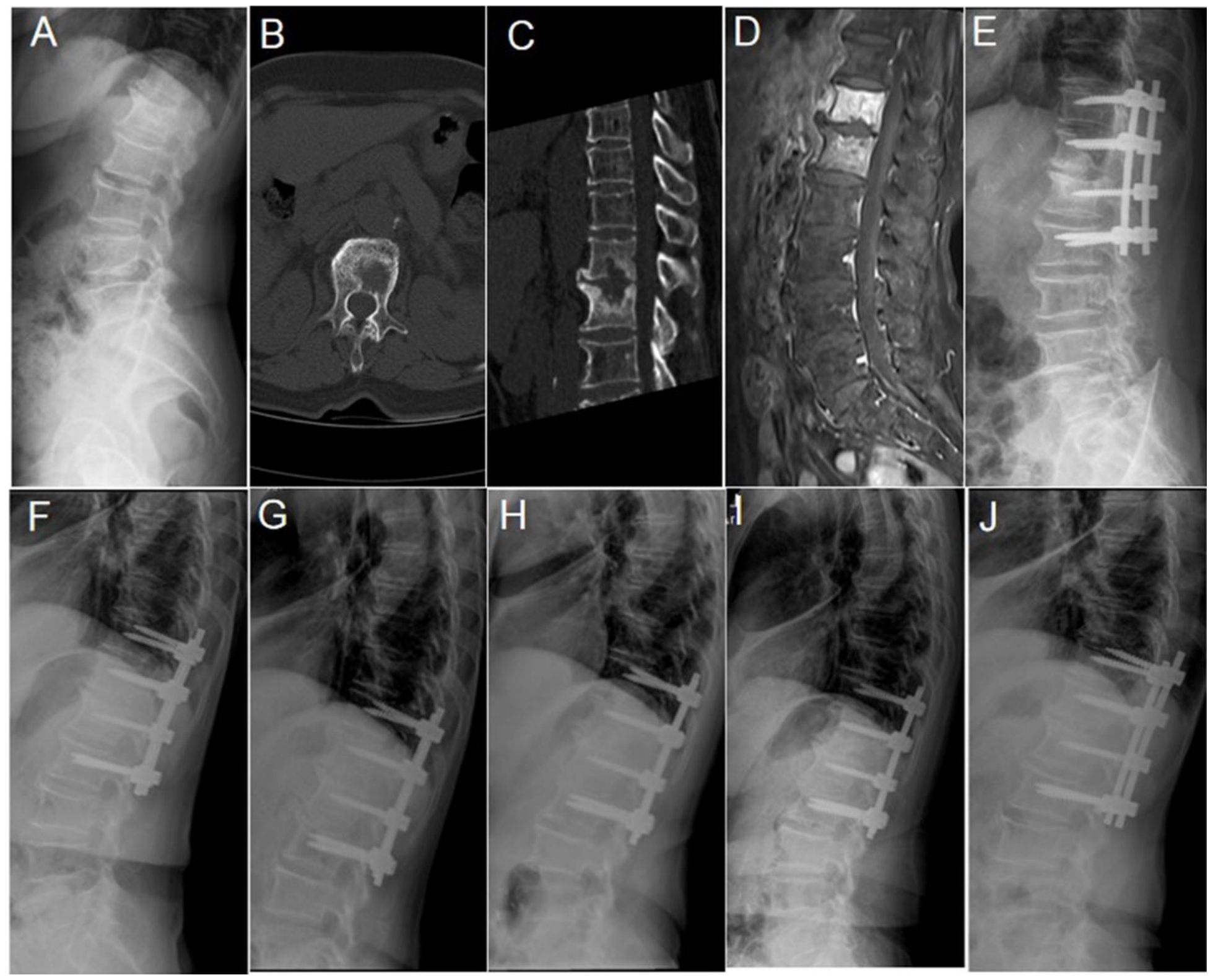

\section{Figure 3}

A 70-year-old woman who experienced pyogenic infection. An x-ray revealed T12-L1 disc space narrowing with end-plate destruction (a); Computed tomography and magnetic resonance imaging (b-d) show vertebral bone destruction and paravertebral abscess formation. X-rays taken at three days, three months, six months, one year, and two years post-operatively, and at the final follow up, respectively (e-j) 


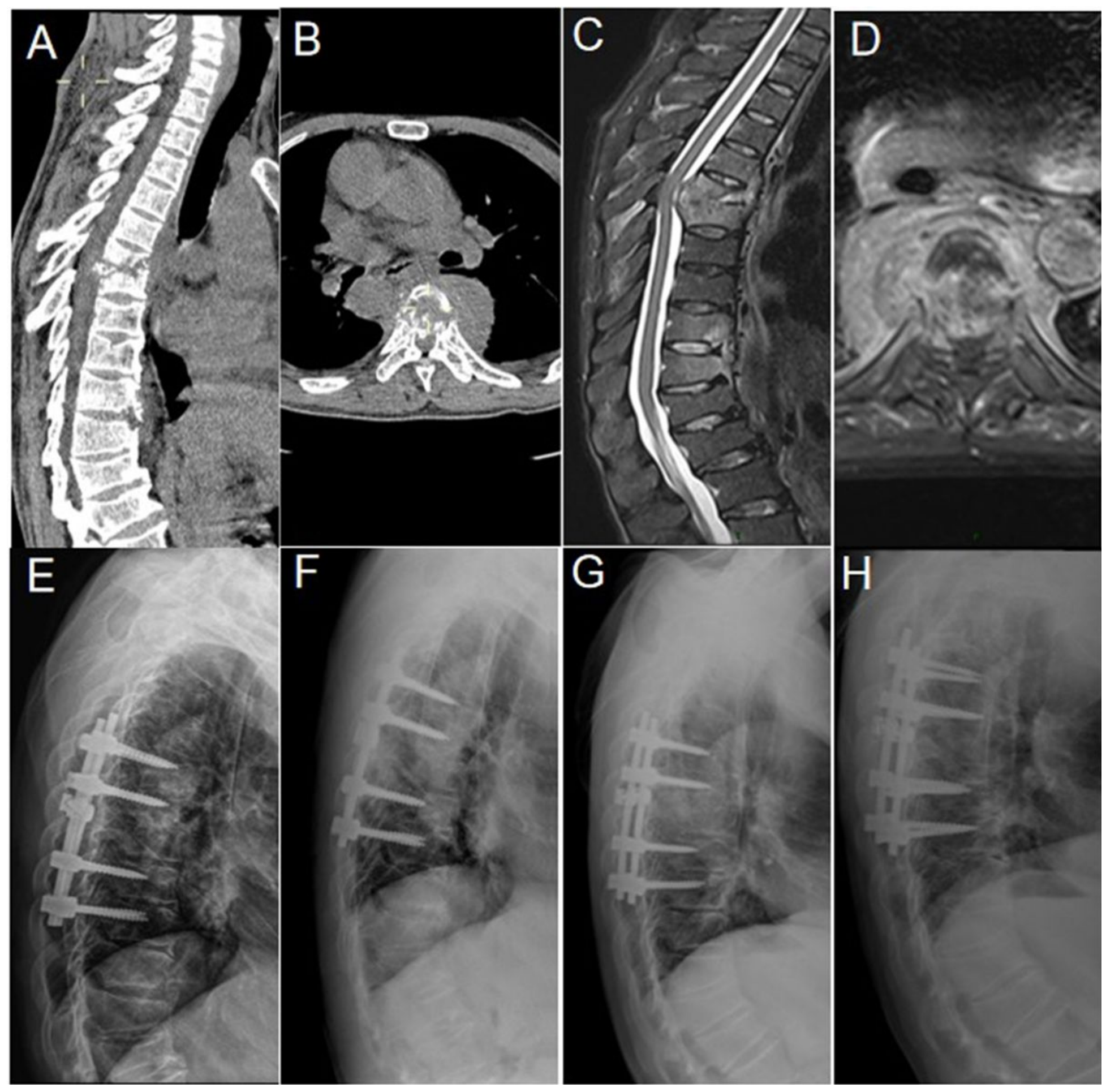

Figure 4

A 67-year-old man with a tuberculosis infection and associated neurological deficit. Computed tomography and magnetic resonance imaging (a-d) show T6-T7 vertebral bone destruction, paravertebral abscess formation and spinal cord compression. X-rays taken three days post-operatively (e); six months post-operatively (f); 12 months post-operatively (g), and; 24 months post-operatively (h) 